\title{
Cancer treatment of today in view of the Nobel Prize
}

The idea that we can activate our immune system to attack cancer is old. Already in 1808, personal physician to Louis XVIII, Dr Alibert, tried to vaccinate himself against cancer by injecting tumour tissue. At the end of the nineteenth century, the surgeon William Coley drew the conclusion from a sarcoma case that infections can counteract cancer by eliciting an immune response and started to treat cancer patients with streptococci (1). Later observations-such as the notion that tumours in rare instances may undergo spontaneous regression, that immunosuppressant drugs increase the risk for cancer, and that the infiltration of $\mathrm{T}$ cells in the tumour goes with a better prognosis-have also indicated the important role of the immune system in cancer. However, it is not until recently that immunotherapy was established as a main treatment for cancer. Indeed, research has increased our knowledge of the immune system and led to better techniques, not least for antibody production.

The Nobel Prize in Medicine 2018 is shared between James Allison and Tasuku Honjo. Checkpoint antibodies, which bind to and block inhibitory proteins on the T cells, have in a few years become an established treatment for several cancer types. Allison's research has paved the way in making the first checkpoint antibody reaching the clinic, and Honjo's research has been decisive in the development of the most commonly used checkpoint antibodies. Also this year's Nobel Prize in Chemistry rewards discoveries that have been of great importance for the development of antibodies. George P. Smith and Greg Winter share the Nobel Prize in Chemistry for improving the manufacturing of antibodies through 'phage display'. This technique means that virus infecting bacteria, bacteriophages, can be utilized to develop new proteins such as antibodies (2). Winter has also developed a technique to manufacture antibodies with less mouse protein, yielding humanized antibodies. The advantages of humanized and human (no mouse protein) antibodies are that the risk for acute reaction decreases and, since they are not degraded as quickly as chimeric antibodies, their half-lives are longer.

Since a couple of decades antibodies are routinely used in cancer care, and new antibodies have steadily been introduced. Most of them are antagonistic (blocking), non-conjugated (not coupled, naked), but a few are conjugated to a toxic substance or a radioactive compound. The majority of them are also monospecific, i.e. binding with their two arms to the same structure and predominantly to a tumour-associated antigen on the tumour cell. Unconjugated antibodies can kill cancer cells in several ways. They are, after binding to the tumour cell, able to affect intracellular signal pathways and thereby induce the cell to undergo apoptosis. Additionally, after binding to the tumour cell, they can by their constant parts activate receptors on immune cells, receptors on immune cells, mostly NK cells which kill the cancer cell. This mechanism is called antibody-dependent cellular toxicity (ADCC). Furthermore, antibodies having bound to cancer cells can activate the complement system via the classical pathway. A membrane attack complex is formed, making a hole in the cancer cell, and death is followed by complement-dependent cytotoxicity (CDC) through osmosis. Yet another mechanism enhancing the anti-tumour effect is that the number of antibodies can increase by the idiotypic network. Bevacizumab, which before the introduction of the checkpoint inhibitors has been the antibody used in most cancer types, does not bind to cancer cells but to VEGF-A. Thereby, the binding of VEGF-A to VEGFR1 and VEGFR2 is inhibited, reducing angiogenesis in the tumour and consequently tumour growth. Examples of bispecific antibodies are antibodies binding with one arm to CD3 and with the other to a tumour-associated antigen. Since $C D 3$ is found on all T cells, these antibodies can join the cancer cells with $T$ cells, resulting in tumour death.

Treatment with checkpoint antibodies is recognized as specific immunotherapy even though they activate the immune system in an unspecific way. The general activation means a risk for autoimmune reactions, and the most common side effects are fatigue, colitis (diarrhoea as cardinal symptom), and skin reaction (dryness, pruritus, and exanthema). In some circumstances it is preferable to give an extra powerful treatment with two types of antibodies. It has been shown that the treatment benefit with this combination immunotherapy is greater for patients who need to stop the treatment due to side effects. If the side effects become severe, treatment with steroids is needed. It is, however, important to avoid steroid treatment as much as possible, since the anti-tumour effect otherwise may be hampered (3). The blocking of inhibitory signals in the immune system by the checkpoint antibodies leads to an activation of cytotoxic T cells. These T cells release perforin, which makes holes in the cancer cell, and granzymes entering the cell through these holes activate the caspase chain leading to cell death. The $T$ cells themselves do not die by the attack but can carry on as serial killers of cancer cells. Tumour types with many mutations, such as malignant melanoma, lung cancer, and urinary bladder cancer, respond better to treatment with checkpoint antibodies. Mutations make the cell express more foreign structures, facilitating the break of tolerance to mount an effective immune response. When treating with chemotherapy, the cancer sooner or later always becomes resistant, but with the introduction of checkpoint inhibitors an increasing number of patients respond completely, i.e. complete remission is achieved. Several of these patients are most likely cured, which is fantastic news in each single case. 
As for other treatments within the field of oncology, a lot of effort has been made to find predictive markers for selecting the right patients for the treatment. In some cancer types the checkpoint inhibitors are, according to study results, used only in patients exhibiting a certain amount of PDL1 in their tumours. Since the most commonly used checkpoint inhibitors block the binding to PDL1, it is logical that a high expression of this protein yields a better response. Interestingly, there are reports indicating that intestinal bacteria are of importance for how well patients respond to treatment with checkpoint inhibitors, and studies are underway to change the microbiome of the patients to increase the response rate (4).

Despite the above-described advances, so far most cancer patients do not benefit from immunotherapy. However, the development in the field continues rapidly, and the treatment will soon be used routinely also in the adjuvant situation in malignant melanoma patients (5). Other important lines of research are combinations of different immunotherapies (for example vaccine + checkpoint antibody) and immunotherapy preceded by chemotherapy, which is called conditioning. To succeed with conditioning, it is important to choose the right chemotherapy in the correct dosage, and the timing is also essential. Conditioning is a way to make 'cold' tumours 'hot', i.e. to make them immunogenic and thereby responsive to immunotherapy. Our most common cancer type, prostate cancer, is one example of a type we hitherto have not succeeded in treating with immunotherapy, and we need to try to make this cancer 'hot'. Antibodies are usually administered intravenously, but in recent years some antibodies have been registered for subcutaneous delivery, which facilitates delivery for both patients and health care. Of note, it is not easy to launch a competitor when the patent for an established antibody expires. Contrary to pills which can be synthesized, antibodies are manufactured in biological systems and are categorized as biosimilars. Hence, new studies are needed in man to prove acceptable toxicity and effect before a competitor is allowed for usage.

We must not forget that there are other important immunotherapies that are used routinely for cancer. The most powerful immunotherapy is to change immune system, and allogeneic stem cell transplantation is a main treatment in the field of leukaemia. Another type of successful immunotherapy used frequently for decades is that of BCG vaccination of superficial bladder cancer patients. Another important immunotherapy is treatment with autologous $T$ cells for malignant melanoma, although it has not as yet become part of routine practice. Moreover, CAR T cells have been registered for treatment of lymphoma patients (6). CAR $T$ cells are $T$ cells genetically changed to produce a chimeric antigen receptor (CAR) partly consisting of an antibody and in part of the enhanced signal domains of the $T$ cell receptor. Thus, CAR T cells can, quite in contrast to normal T cells without antigen presentation, become activated to kill tumour cells. A hot research field is immunostimulating gene therapy (7). For example, a study with a virus acting as a vector for immune-stimulating genes is ongoing in Uppsala. The virus is injected repeatedly intratumourally and cancer cells are destroyed (oncolysis), hopefully stimulating an immune response against the cancer that also is effective in noninjected metastases.

The best is of course not having to get cancer treatment at all, and the cancer type with the fastest-growing incidence in recent decades is malignant melanoma. The advice is therefore not to go to Thailand. In addition to a decrease in the risk for developing malignant melanoma, the likelihood for future patients to receive the new immunotherapy increases. The reason is that there is a risk that carbon dioxide emissions might disable modern health care, which is a prerequisite for advanced cancer treatment.

In conclusion, in recent years immunotherapy has-in addition to surgery, radiotherapy, and chemotherapyemerged as the fourth cornerstone in cancer treatment. In view of the fact that almost 100,000 Swedes will be diagnosed with cancer in 2040 according to the Swedish Cancer Society, the discoveries behind the Nobel Prize in Medicine 2018 are particularly appreciated. At the same time, it is of utmost importance to continue our efforts in the field of research with the vision that all who are struck with this widespread disease will be cured.

\section{References}

1. McCarthy EF. The toxins of William B. Coley and the treatment of bone and soft-tissue sarcomas. lowa Orthop J. 2006;26:154-8.

2. Winter G, Griffiths AD, Hawkins RE, Hoogenboom HR. Making antibodies by phage display technology. Annu Rev Immunol. 1994;12: 433-55.

3. Brahmer JR, Lacchetti C, Schneider BJ, Atkins MB, Brassil KJ, Caterino JM, et al. Management of immune-related adverse events in patients treated with immune checkpoint inhibitor therapy: American Society of Clinical Oncology Clinical Practice Guideline. J Clin Oncol. 2018;36:1714-68.

4. Routy B, Le Chatelier E, Derosa L, Duong CPM, Alou MT, Daillere R, et al. Gut microbiome influences efficacy of PD-1-based immunotherapy against epithelial tumors. Science. 2018;359:91-7.

5. Weber J, Mandala M, Del Vecchio M, Gogas HJ, Arance AM, Cowey $\mathrm{CL}$, et al. Adjuvant nivolumab versus ipilimumab in resected stage III or IV melanoma. N Engl J Med. 2017;377:1824-35.

6. June $\mathrm{CH}, \mathrm{O}^{\prime}$ Connor RS, Kawalekar OU, Ghassemi S, Milone MC. CAR T cell immunotherapy for human cancer. Science. 2018;359:1361-5.

7. Loskog A, Maleka A, Mangsbo S, Svensson E, Lundberg C, Nilsson A, et al. Immunostimulatory AdCD40L gene therapy combined with low-dose cyclophosphamide in metastatic melanoma patients. $\mathrm{Br} \mathrm{J}$ Cancer. 2016;114:872-80.

Gustav Ullenhag

Scientific Secretary, Swedish Society of Oncology Associate Professor and Consultant in Oncology, Uppsala University Hospital 